\title{
A Constant Threshold based Clustering using Fuzzy Inference in Wireless Sensor Network
}

\author{
Yogender Kumar Sharma \\ M. Tech., Department of CSE \\ Keystone Group of Institutions \\ Pilod(Surajgarh), Jhunjhunu - 333029
}

\author{
Rajveer Singh \\ Assistant Professor, CSE \\ Keystone Group of Institutions \\ Pilod(Surajgarh), Jhunjhunu - 333029
}

\begin{abstract}
Wireless Sensor Network (WSN)is a network containing insignificant sensors node(SN) through low electrical transducers used as a data collecting tool in various environments based on the network setup. Designed message or communication passing procedure designed to protect the incomplete dynamism properties of sensors aimed at data processing is the main task of WSN. To accomplish this task, we are introducing a new concept for saving energy and enhancing the Network's network length. Clustering is innumerable in areas where excluding clustering
\end{abstract}

Each compartment uses a different algorithm to utilize multi-hip routing, using an appropriate nod code to conduct information from the base station, from every position to every round. The residual energy, no. of nodes, distance within every node is estimated such as the Fuzzy criterion for selecting cluster head FMCR-CT, network lifetime, dead rounds in every round, first node decease, half node dies as well as the previous node dies.

A sensor network can be made scalable by forming clusters.

A sensor network can be made scalable by forming clusters.

A sensor network can be made scalable by forming clusters.

A sensor network can be made scalable by forming clusters.

A sensor network can be made scalable by forming clusters.

A sensor network can be made scalable by forming clusters

\section{Keywords}

Crime Analysis, Criminology, Data Mining. Crime Prediction

\section{INTRODUCTION}

The WSN is the maximum sensor of the network to monitor physical otherwise ecological conditions. Generally, a WSN is an $\mathrm{n} / \mathrm{w}$ of protuberances that collaborate now understanding as well as exploring the environment around them. Sensor nodes, each node connected to each other. Communication components include a radio receiver, microcontroller and power sources include sensor nodes. They combine the process of data collection together. [2] [3], communicates with short-distance sensor nodes via the Wireless Communication medium.
The WSN is now widely used in most applications since the Internet of Things (IoT) has been expanded into Information Communications Technology (ICT). Owing to the environment of the device protuberance centered on the process to collect as well as send data based on the $\mathrm{n} / \mathrm{w}$ arrangement, power is an important issue on WSN. The manuscripts emphasize the power supply of batteries applied to rule the device protuberances.

Sequences are the key basis of electricity aimed at the device protuberance, making sure that $\mathrm{n} / \mathrm{w}$ message is tied to the network. Removable and non-rechargeable batteries are described in the lifestyle, which requires changing or recharging to make energy available.

In some WSN Topology networks, it is difficult for other users to have their own batteries easily changed, charged or recharging due to the behavior of the topology setup network difficulty. Network topologies, such as hydroelectric networks or mining, conveys, forests or other dangerous places for humans.

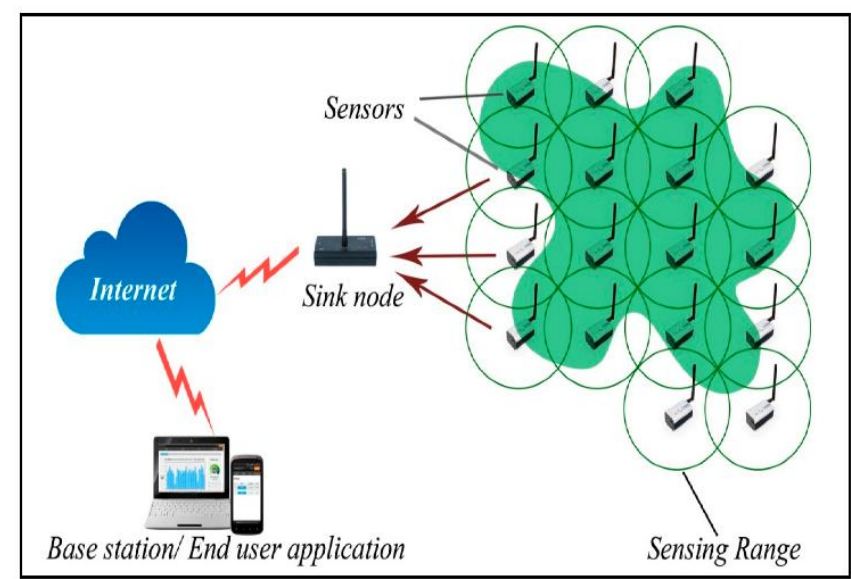

Figure 1 Wireless Sensor network

The sensor network has numerous experiments when associated with the local area network (LAN) or the parallel systems like the WAN. The traditional $\mathrm{n} / \mathrm{w}$ has some physical nodes, communication broadcasting, switch, rounder's, as well as other system strategies, which are like an Ad-hoc network when compared to WSNs. As data shows, WSNs construction for WSN's data dispensation as well as the network environment.

Moreover, the batteries do not only have different transitions, which also influence the images of transmission media, network topology setup, as well as bandwidth topic [4]. 


\section{RELATED WORK}

Energy conservation is a major problem when WSN is designed. Therefore, the sensor nodes, designed to run with battery power and other materials, from a national grid power system to a source. Investigators in the field [9] are investigating the possibility of closing the power consumption on battery, hardware or procedure stack levels. Many network routing protocols have been suggested for virus networks checked in the background of WSNs constructed on dynamism efficacy to confirm that $\mathrm{n} / \mathrm{w}$ is always dynamic. Felicia [11] encourages long-term sensor nodes to make the operating system more efficient and efficient. Felicia's idea [11] helps, but battery life is a few challenges.

Lee at el [12] Presents the challenge to live communication aimed at WSNs. Computing power besides narrow bandwidth delivers uninterrupted interruptions with live communication. Thus, subjects, as well as research challenges, consume addressed to live message on WSNs.

Kapalua at el [13] The clustering method has been suggested to enhance the network's lifespan. The process to be implemented over time. Each round is divided into two stages: Cluster Building Stage, Stable Data Daily Commissions Step [14]. However, Rezaei and Mobininejad [15] identified two major product networks: Duty cycling, and the neutral approaches to data to ensure energy without wasting energy. Not all of the specific texts can have a fixed fix since all sensor nodes are based on the battery chemistry.

Pubill at el [16] Suggest energy harvesting by artificial lighting. A WSN consists of data that is sent to the energy harvesting component of the proposed solution in the source node of a WSN. Photovoltaic harvesting artificial light from the nearest lamp with an energy harvesting module and PV (PV) cell contains. Energy WSN node features will be added was changed to the correct power supply, $3 \mathrm{~V}$ coin battery can be rechargeable, electric energy.

Idrees et al. (2016) A SN developed by Molenet to grip wireless substitute process. The system is a share of the revival campaign in Cameroon. Their chief objective is to operate the system aimed at extended hours as well as increase the level of message [16].
Davide et al. (2009) A water WSN is centered on optical interaction amongst nodes. Radio occurrences, as well as sound waves, are great in aquatic. To incredulous this problematic, optical message can be measured. To increase the competence of the scheme, an operator interface was designed to perform Optical Physical (PHY) layer. Digilent Spartan third Board [19].

Nithin et al. (2017) The primary methods as well as problems connecting to a quest for study have been reviewed. A broadside was constructed on a review of a cellar centered on a study of the underwater cellular sensor network(SN) through highlighting its compensations as well as advantages. Authors implement different clustered techniques to implement data aggregation to validate the impact of information compilation [20].

\section{FUZZY LOGIC}

Fuzzy refers to things that are meaningless or unclear. In the real world most often in the country is the truth or wrong, and their moral logic philosophy is very valuable. In this case, let us consider the precision and uncertainties of any circumstances.

- RULE BASE: On the basis of language information, experts from experts offer a set of rules and sectors that provide. Current developments in Fascist theory provide many effective methods for designing and tuning of Fuzzy Controllers. Record of these changes decreases the sum of obscure laws.

- FUZZIFICATION: Instead of converting inputs, change sensational numbers. Spirit inputs are basically calculated by sensors to measure precise inputs, as temperature, pressure, rpm's, etc.

- INFERENCE ENGINE: Controls the corresponding physique of the existing physiological contribution which is in accordance with each law, and decides which rules must be avoided by the input field. Next, regulated laws will be added to form controlled activities.

- DEFUZZIFICATION: It is used to change the referential set obtained as the appropriate value for the reference engine. There are several depth modes for reducing the errors and utilizing a well-versatile specialized system.

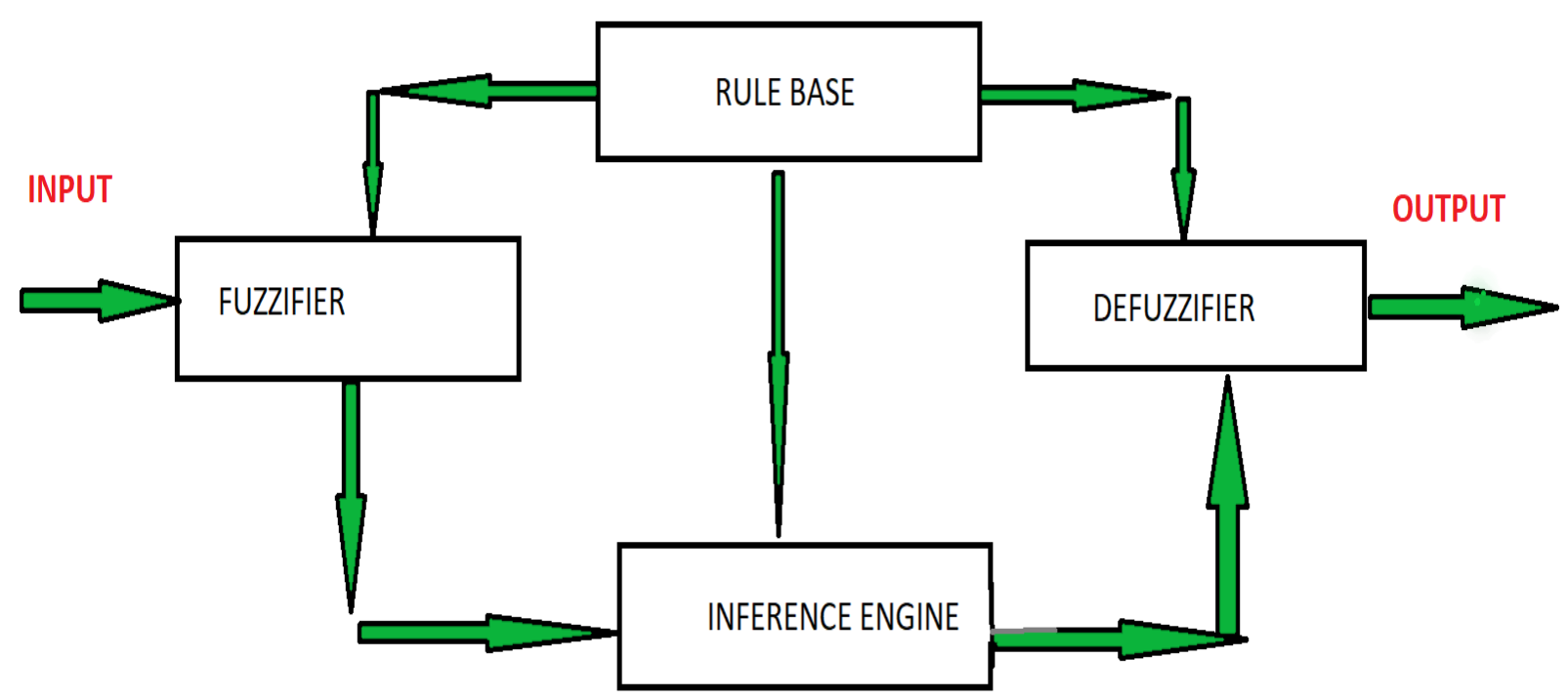

Figure 2 Fuzzy System 


\section{METHODOLOGY}

The point of the suggested algo is towards increasing the lifespan of remote sensor arranges through decreasing the number sent messages. It is accomplished through thinking about a consistent edge, utilizing distinctive bunching strategies in each round \& exhibiting a multi-jump way to deal with transfer the information towards the base station (BS). Common highlights of the suggested algo are such per the following:

Non-random collection of $\mathbf{C H}$ : Now FMCR-CT, $\mathrm{CH}$ node is designated depend upon Fuzzy Logic into the disseminated method.

Various clustering method in every round: To do it, each circle of bunch heads is done by a variety of methods. In the main group, the group head hub determination depends on the "residual living" \& "thickness" of each hub. Growing to reduce the amount of sent messages \& organize life, without any determination in the second group, the group of the last round is the $\mathrm{CH}$. In the $3^{\text {rd }}$ bunch, group head purpose is completed dependent proceeding the "measure of remaining vitality" \& the "separation of the hub towards the past bunch head hub".

Unequal clustering: Inequality groups are acquired into the suggested Suzanne-FMCR-CT calculations. The amount of the group is resolute through the separation from the BS. Next, to these lines, the hub gets more remote from the base station, the larger the hive. The point of with the inadequate group into this calculation is towards decrease the hotspot issue on the remote sensor system.

Determining fixed threshold: FMCR-CT efforts to decrease controlled communications \& prolongs the $\mathrm{n} / \mathrm{w}$ life span with the permanent threshold. Distributable method: In FMCR-CT, each $\mathrm{n} / \mathrm{w}$ performance for example clustering, $\mathrm{CH}$ node selection and routing based station at BS is run through $\mathrm{n} / \mathrm{w}$ nodes deprived of intrusion.

Multi-hop data transmitting: The effort to improve the system exhibit by launching multi-jumping steering outside of this work bunch. Data is collected through a multi-bounce system from each group to a single consolidation \& after every bunch towards the BS and it ends with selecting the right-center reliant on the "separation after the BS" \& the 'amount of residual energy'

As described formerly, the main goal of clustering on WSN is to reduce the power consumption of the system. Though, from one node to another, the others repeatedly replicated the energy that they were doing on or off the base station. Various methods have been offered. This article is useful for carrying out the node clustering procedures on the system. Introducing a cluster-based routing system. This includes the number of cluster headgear selections, frequent messaging, and guarantee for the multi-hop routing protocol. Increase the ability to save energy on the $\mathrm{n} / \mathrm{w}$.

\section{System model}

FMCR-CT algorithm includes the concept:

- All nodes are combined with equal primary power

- Randomly distributed Nodes into the $\mathrm{n} / \mathrm{w}$.

- $\quad$ The BS \& nodes are static.

- To calculate distance Euclidian technique is utilized.

- $\quad$ Base Station Data Transmission Multi-Hop \& Specific Events, Solitary Hop.

- Protuberances in $\mathrm{d}$ after a definite node are deliberated such as neighbors of its protuberance.

The energy prototypical used to refer the L-bit packet information as of the receiver is located between $d$ to the distance amongst each other, as follows:

$E_{T X}(l, d)=\left\{\begin{array}{l}l * E_{\text {elec }}+l * \varepsilon_{f s} * d^{2} \text { ifd }<d_{0} \\ l * E_{\text {elec }}+l * \varepsilon_{m p} * d^{4} \text { if } d>d_{0}\end{array}\right.$

Where $d_{0}$ the threshold (d) to define uncertainty transmission model is a unrestricted interplanetary or multipath promotion model. Uncertainty space among sender \& the receiver node is less than $\mathrm{d}$, the transmission model is unrestricted as well as uncertainty it is above 20 before the transmission model is multipath promotion. $d_{0}$ Is derived after the succeeding relation:

$$
d_{0}=\sqrt{\frac{\varepsilon_{f s}}{\varepsilon_{m p}}}
$$

$E_{\text {elec }}$ is considered as the consuming energy degree for transmitting data to each bit from the receiver to the receiver. The $\mathrm{e}_{\mathrm{fs}}$ represent the erosional energy degree to send data into open the air, \& emp the consuming energy essential aimed at multipath expansion. ERX denotes the power essential towards obtain information through receivers obtained from the following relationships:

$$
E_{R X}=l * E_{\text {elec }}
$$

As mentioned above, by reducing the number of combinations, the FMRR-CT algorithm aims at plummeting the numeral of messages sent to the network's lifetime. Thus, in this paper, 3, unlike algae clusters, are applied to cluster nodes as well as to choose the furthermost effectual at cluster level with the help of Fuzzy meridian to advance in the clustering procedure. In this unit, the position of clustering for cluster head selection as well as their direct effect on the communal system gathering.

- Residual energy: because of the significance of $\mathrm{CH}$ work into every cluster, the remaining energy is an opportunity to developing the remaining $\mathrm{CH}$.

- The density of the node: As neighboring node no. play a fundamental role in reducing the energy costs of nodes, this restriction is utilized as one of the standards aimed at $\mathrm{CH}$ collection.

- The node's space towards the earlier round's CH: Due to the importance of the position of the node of each cluster, this restriction is deliberated like the criterion for the $\mathrm{CH}$ collection procedure. So, there is a good chance of selecting the $\mathrm{CH}$ of the node with a short distance from the previous round cluster head.

\section{THE PROPOSED METHODOLOGY SUGENO FUZZY INFERENCE SYSTEM}

As declared earlier, reducing energy costs among networks with the main goal of clustering on wireless sensor networks. However, repeatedly sending messages from one node towards any other or from the BS will reduce the power. Several methods of focus on how to increase energy savings in networks are proposed. In this article, Sugeno fuzzy has an argument for running node clustering procedures on the $n / w$, which grants a cluster-based routing technique, which decreases the no. of $\mathrm{CH}$ selections, decreases the frequency of messages, \& increases the power saving power in a multi-hop network, while routing protocols.

Benefits of the Sugeno Systems:

- Computational flexibility. 
- Exertion well through linear methods like PID control

- Exertion well through optimization as well as adaptive methods.

- Output Surface Guarantee Permanency.

- Suitable for mathematical analysis threshold

1. $\mathrm{n}=$ no. of rounds

2. $\mathrm{r}=$ current $\operatorname{round}(\mathrm{CR})$

3. Round $=1$

4. $\mathrm{Th}=0.7$

5. neighbors $(i)=$ set of nodes that are neighbors of node $\mathrm{i}$

6. neighbors Counter(i) $=$ no. of neighbors $(i)$

7. $\quad$ energy $(i)=$ the remaining energy of node $\mathrm{i}$

8. $\mathrm{CH}(\mathrm{i})=$ cluster head of node $\mathrm{i}$

9. Criterion $(\mathrm{i})=$ efficiency value of $\mathrm{CH}$

10. if $($ Round $=1)$ then

11. used for every integer $i$ in $n$ do

12. $\operatorname{chance}(\mathrm{i})=$ fuzzy $($ energy $(\mathrm{i})$, neighbors Counter(i))

13. transfer (chance(i), neighbors(i))

14. $\mathrm{CH}(\mathrm{i})=\operatorname{best}($ chance $)$

15. transfer(data(i), $\mathrm{CH}(\mathrm{i}))$

16. $\operatorname{en}(i)=\operatorname{energy}(i)$

17. end for

18. Round $=2$

19. else if (Round $=2$ ) then

20. used for every integer $i$ in $n$ do

21. if ( $\mathrm{CH}(\mathrm{i})$ is alive) then

22. transfer (data(i), $\mathrm{CH}(\mathrm{i})$ )

23. Else

24. $\mathrm{CH}(\mathrm{i})=$ best $($ chance $)$

25. transfer (data(i), $\mathrm{CH}(\mathrm{i}))$

26. end if

27. if $(\operatorname{energy}(\mathrm{CH}(\mathrm{i})>\operatorname{th} * \operatorname{en}(\mathrm{CH}(\mathrm{i})))$ then

28. Round $=2$

29. Else

30. Round $=3$

31. end if

32. end for

33. else if ( Round $=3$ ) then

34. DistanceToCH(i) = calculate distance from node $\mathrm{i}$ to $\mathrm{CH}(\mathrm{i})$

35. used for every integer $i$ in $n$ do

36. chance $(\mathrm{i})=$ fuzzy $($ energy $(\mathrm{i})$, DistanceToCH(i)
37. transfer (chance(i), neighbors(i))

38. $\mathrm{CH}(\mathrm{i})=$ best $($ chance $)$

39. transfer (data(i), $\mathrm{CH}(\mathrm{i})$ )

40. end for

41. end if

42. used for every integer $\mathrm{i}$ in $\mathrm{n}$ do

43. Calculate criterion for every $\mathrm{CH}$

44. $\mathrm{SCH}(\mathrm{i})=$ best $($ criterion $)$

45. if $\mathrm{SCH}(\mathrm{i})==0$

46. transfer (data(i),BS)

47. else

48. transfer (data(i),SCH(i))

49. data aggregated by $\mathrm{SCH}(\mathrm{i})$

50. $\operatorname{transfer}($ data(i),BS)

51. end if

52. end for

$1^{\text {st }}$ Clustering Scenario:

In the $1,4,7$ rounds, the capacity of energy, as well as the neighbors of every node, are regarded as vague $\mathrm{I} / \mathrm{p}$.

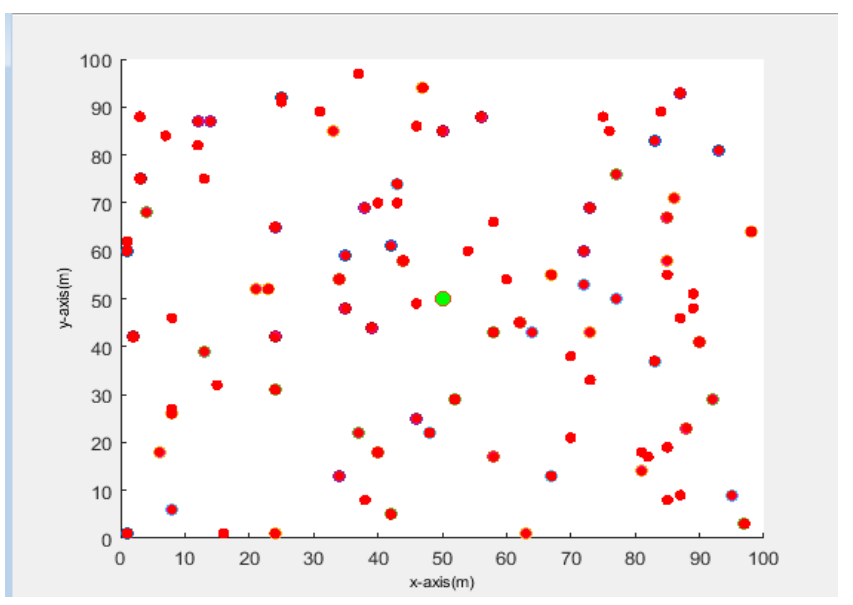

Figure 3 Scenario 1

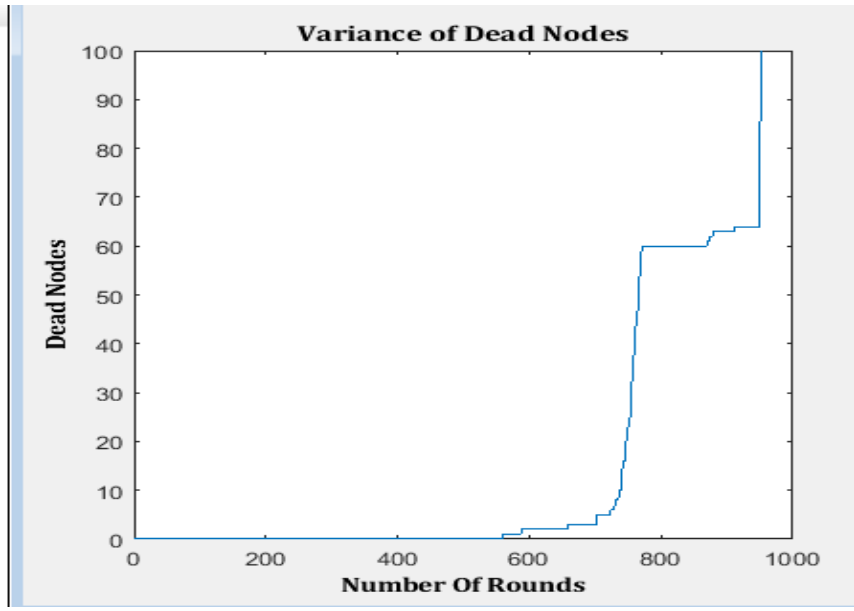

Figure 4 No. of Dead Nodes per Round Scenario. 


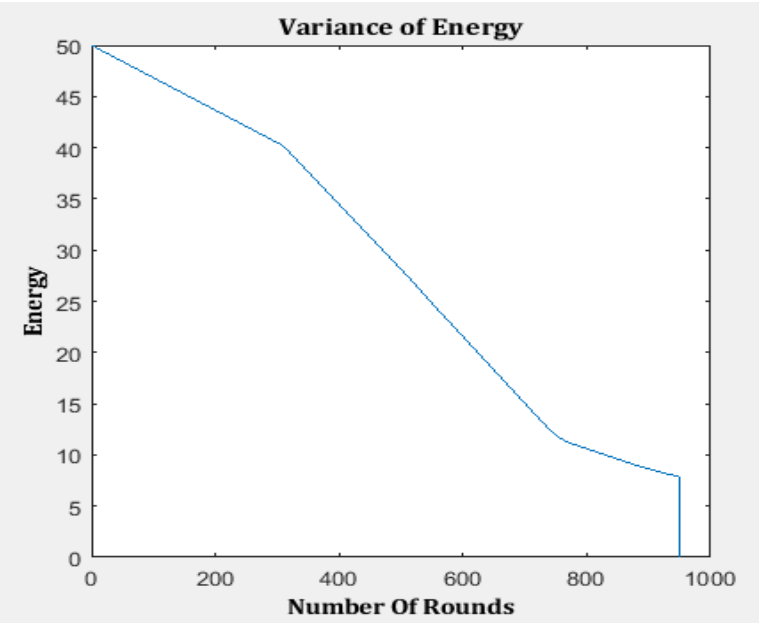

Figure 5 Energy Consumption Rate in Every Round Scenario 1.

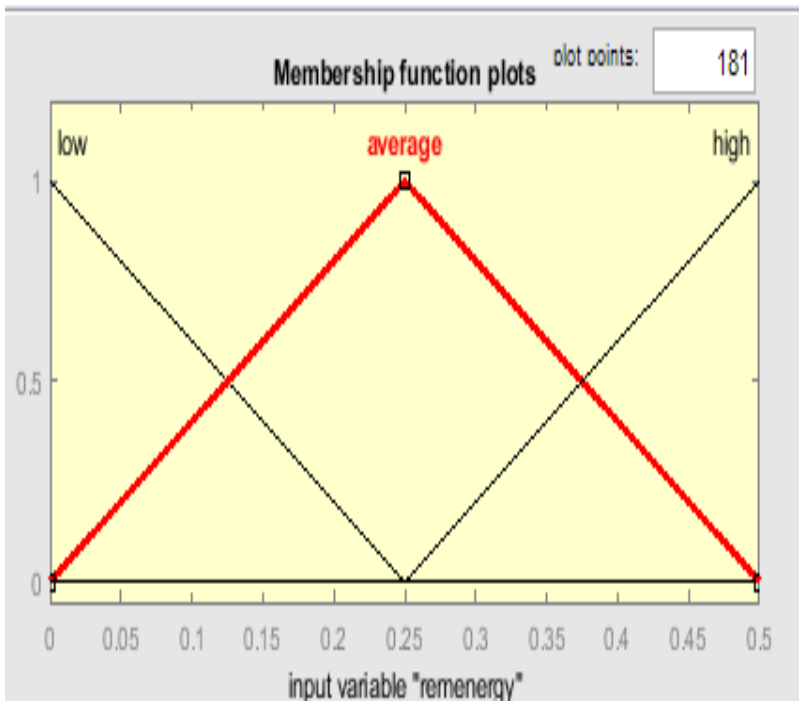

Figure 6 The I / p Subscription Work of T Residual Energy in the First Clustering.

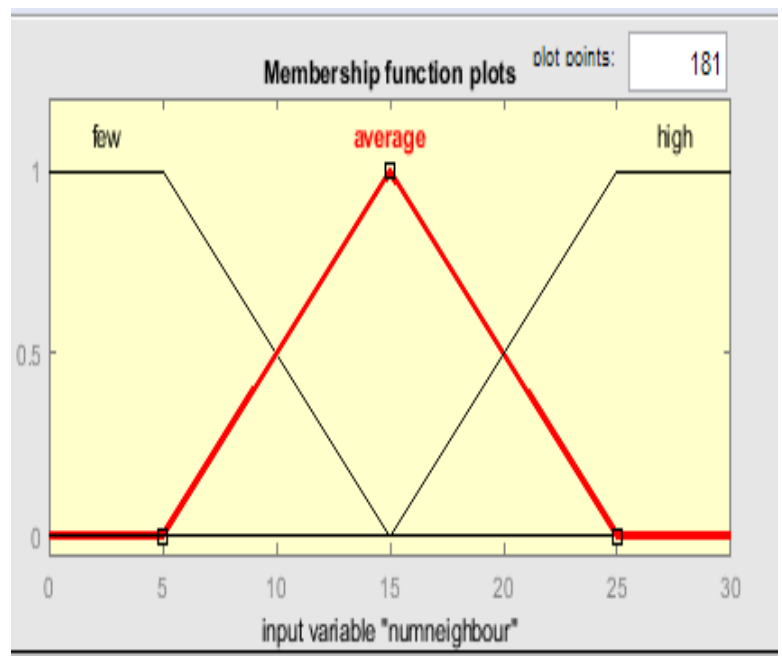

Figure 7 Number of Neighbors in the 1st Cluster is a Membership Function.

Membership functions of 2 parameters are displayed in figures. 6 as well as 7Subsequently assuming the assumption as well as the possibility, every node transmits the production produced toward the neighbors, finally the node through the most productive node at the cluster level of that node. The number of opportunities will be sent to each node from the signal of this signal, and the risk of receiver nodes can be compared to others. At the conclusion of this phase, the node will be the $\mathrm{CH}$ in the neighborhood as well as will transmit a message to all the protuberances inside the signal.

\section{Second scenario clustering}

Subsequently, the node's energy, as well as the number of their neighbors, have not changed much at the conclusion of the first cluster, the $\mathrm{CH}$ of the next round is likely to be reelected in the next round. Therefore, no choice will be in the second cluster. After $\mathrm{CH}$ are first resolute in gathering, certain of their dynamism is kept in a variable (en). Uncertainty the first of all clusters are further dynamism than the significance of significance (variable), they determination be in the second clustering. Then, all $\mathrm{CH}$ determination transfer to third clustering.

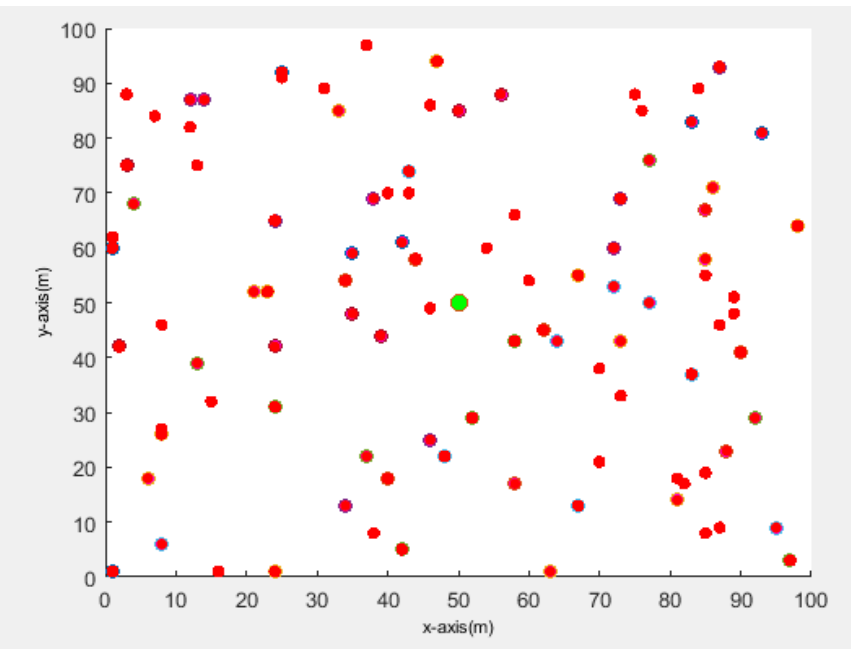

Figure 8 Scenario 2

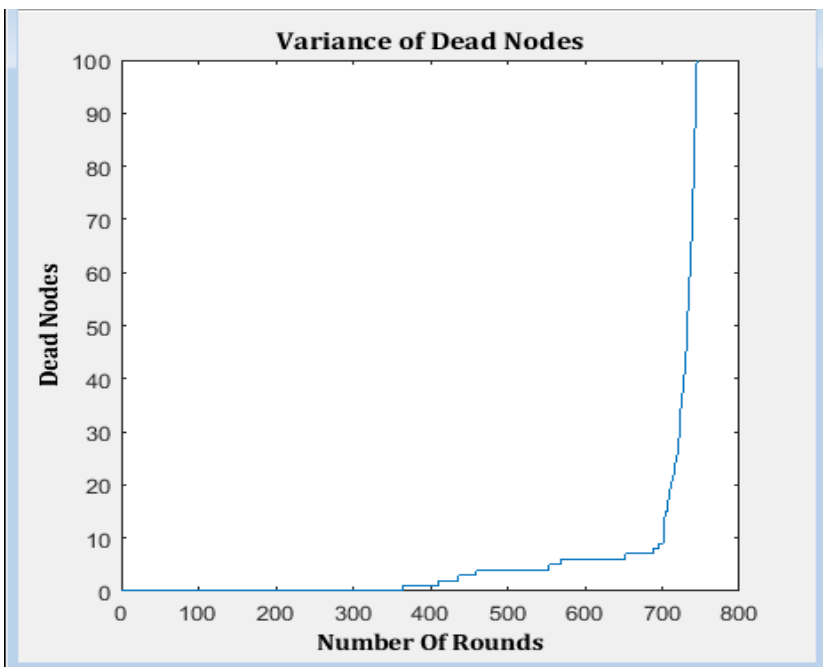

Figure 9 The Number of Dead Cells In Each Side is Scenario 


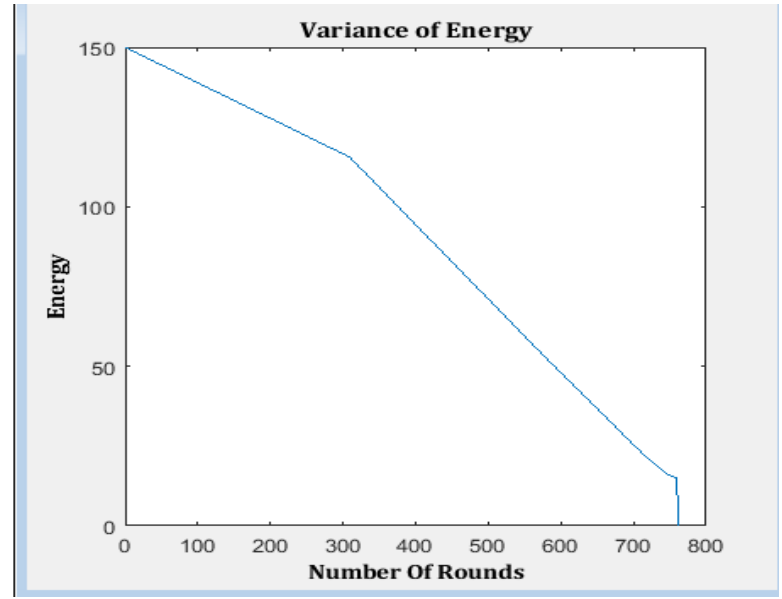

Figure 10 Energy Consumption Rate in Every Round Scenario 2.

\section{Third Clustering Scenario}

Third clustering will be applied in 3, 5 and 7 rounds. Because the nodes are energy sensors, one of the main components, node energy is utilized for example unique of the fuzzy $\mathrm{I} / \mathrm{p}$ strictures of the 3rd cluster. In calculation to the residual energy, the location in every node in the cluster is significant. The space to every node is the 2 nd intermission parameter of the system on the cluster level of the round. Estimates of the blurring rules in this clustering are considered as a value between 0 as well as 1 every node has the option. Every node refers to its unintended to all protuberances, as well as they compare this message with the comparison of others with the opportunity. A node through the highest Node presents itself as a $\mathrm{CH}$ as well as conducts a communication to completely new nodes.

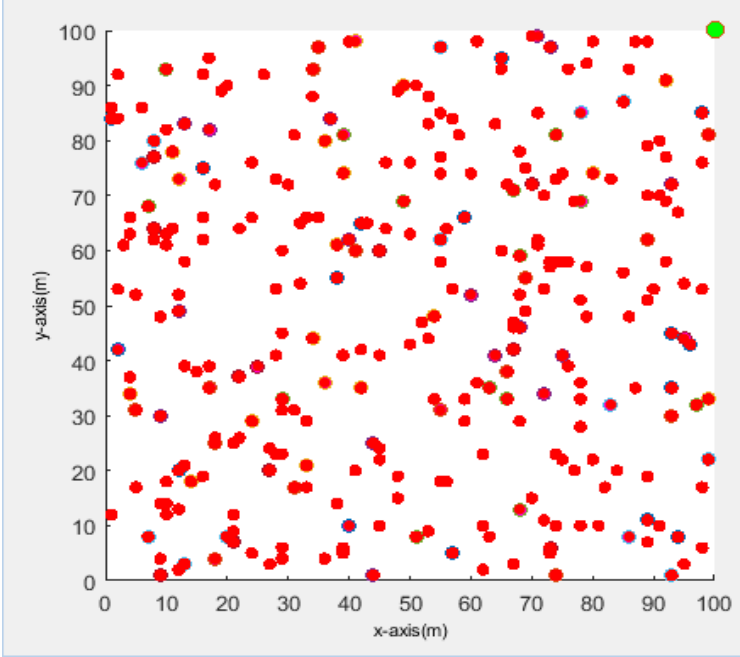

Figure 11 Scenario - 3

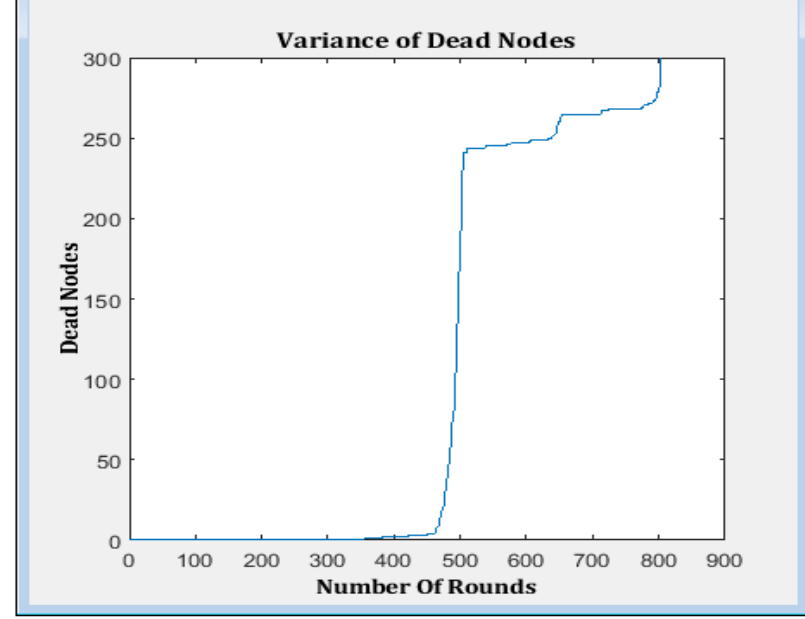

Figure 12 No. of Dead Eggs in Every Round Scenario.

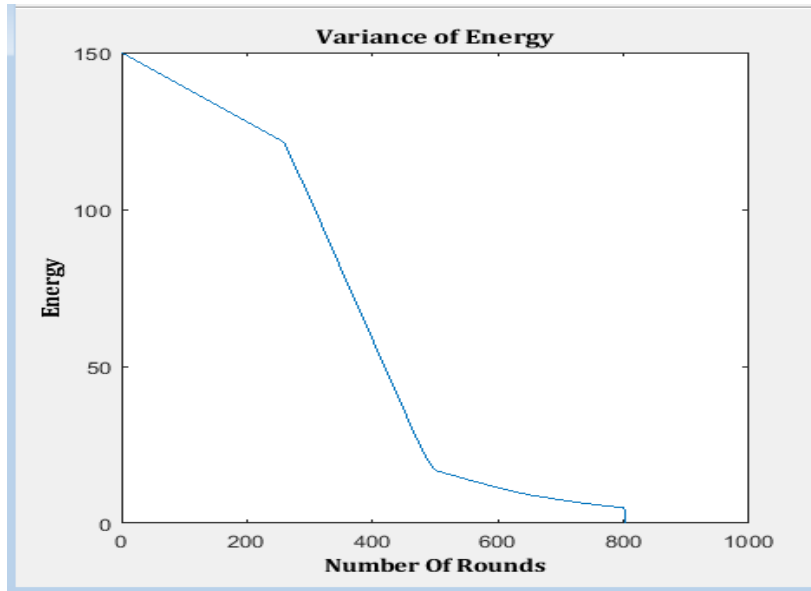

Figure 10 Energy Consumption Rate in Every Round of Scenario 3.

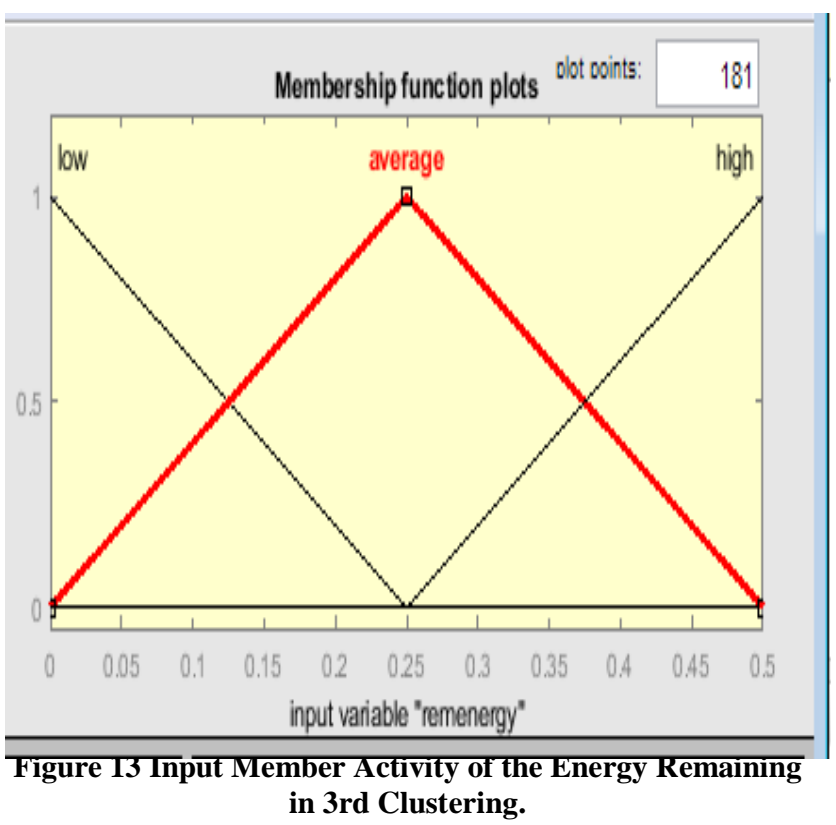




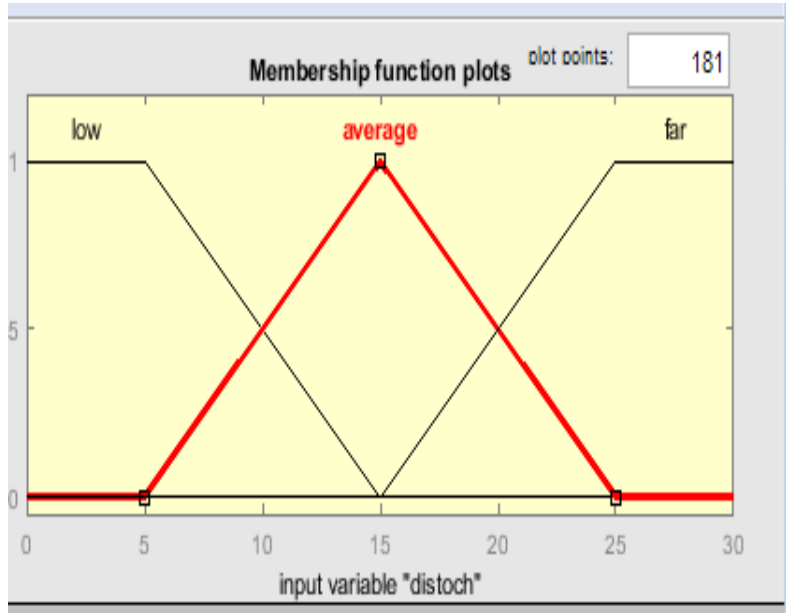

Figure 14 Enter Membership Activities from CH Node and 3rd Customer Round.

The fuzzy effects of this cluster are strictly attainable in the membership work statistics. 13 and also 14The proposed algorithm has been selected as the best node cluster bases in the first clustering network. Believing they will not be a choice in the second clustering. This causes the number of strict messages sent to be reduced. Nets 2, 4 and 6 do not have any clusters, so the designated nodes in the 1 st round will agonize since an absence of energy. Clustering will be complete in the 3rd cluster. The designated cluster mechanism on the 3rd round. This collection is below the inspiration of what's done in the 1 st cluster, not the greatest nodes. Cluster is implemented when the cluster levels are recovered in the first cluster that has lost more than one energy. Strict contribution strictures in the first, as well as third clustering, are dissimilar. Based on the above mentioned, energy is considered to play a significant part in wsn; It is also applied in clustering. Furthermore, the no. of neighbors is enormous. As a consequence, the optimal conditions should be based on the selected node energy and physical condition as a cluster head.

\section{ROUTING}

The purpose of designing protocols that are routed through WSN is designed to decrease dynamism ingesting. Selects using a dressed directing approach as well as ideal stages the node's space since the dishonorable position has a significant impact on the network's life as well as its presentation. This station uses a multi-hop method to send the information BS. FMCR-CT also attempts to node the BS through the cluster head leader node, while transferring central ecological information to each node in the FMCR-CT at each round., Uses the multi-hop method. Cluster Head Leader Node (CHLN) has been selected from the designated collection stages centered on the appropriate criteria. Compatibility criteria are the space to BS \& the residual energy. criteria used for $\mathrm{CH}$ lead node criteria is given below:

$$
\text { Criterion }(\mathrm{CH}(i))=\frac{\text { Distance to base stationCH}(i)}{\text { Energy }(\mathrm{CH}(\boldsymbol{i}))}
$$

Contest radius used for selecting leader node Get below the prevailing cluster levels Relationship:

$$
\text { Competition Radius }=\frac{C H(i) \text { Distance to base station }}{2}
$$

\section{CONCLUSION}

In this research paper, we analyzed cluster-based routing algo's using constant thresholds towards conserving further energy by reducing the no. of switch messages as well as extending the generation of the WSN. Sugeno-FMCR-CT uses benefits of clustering as well as multi-stop broadcast instantaneously. In order to analyze scalability for node numbers, the location of the network \& BS position is applied towards four situations. In all cases, the SUGEN-FMCR-CT could decrease the number of controlled messages, improve LND \& HND parameters, \& use different clustering methods, considering constant thresholds, such as avoiding clusters in all rounds, such as taking action by performing network operations, multi-hop routing \& consider an appropriate mid node. Constant Threshold Advanced N/Ws Presentation. As a result, the suggested algo doesn't appreciate the greatest FND. Sugeno-FMCR-CT performs well in terms of performance whenever HND \& LNG work well for big importance. In our next test, Clustering-based routing algo will use variable threshold values, it can decrease motion costs.

\section{REFERENCES}

[1] Q. Gao, D. Holding, Y. Peng, and K. Blow, "Energy efficiency design challenge in sensor networks," in London Communications Symposium, 2002.

[2] S. Ali, A. Ashraf, S. B. Qaisar, M. K. Afridi, H. Saeed, S. Rashid, E. A. Felemban, and A. A. Sheikh, "Simplimote: A wireless sensor network monitoring platform for oil and gas pipelines," IEEE Systems Journal, vol. 12, no. 1, pp. 778789, 2018

[3] F. Engmann, F. A. Katsriku, J.-D. Abdulai, K. S. AduManu, and F. K. Banaszek, "Prolonging the lifetime of wireless sensor networks: A review of current techniques," Wireless Communications and Mobile Computing, vol. 2018, 2018

[4] J. Lee, B. Shah, G. Pau, J. Prieto, and K.-I. Kim, "Real-time communication in wireless sensor networks," Wireless Communications and Mobile Computing, vol. 2018, 2018.

[5] K. Kapalta, R. Singh, and A. Gautam, "Energy-efficient techniques of wireless sensor networks: A review," International Journal of Advanced Research in Computer Engineering \& Technology (IJARCET) Volume 6, Issue 2, February 2017, ISSN: 2278 - 1323,

[6] D. Pubill, J. Serra, and C. Verikoukis, "Harvesting artificial light indoors to power perpetually a wireless sensor network node," in 2018 IEEE 23rd International Workshop on Computer Aided Modeling and Design of Communication Links and Networks (CAMAD). IEEE, 2018, pp.

[7] S. D. Attri and A. Tyagi, "Climate profile of India," Environment Monitoring and Research Centre, India Meteorological Department, Lodi Road, New Delhi110003 (India), 2010

[8] C. Schurgers and M. B. Srivastava, "Energy efficient routing in wireless sensor networks," in Military communications conference, 2001. MILCOM 2001 Communications for network-centric operations: Creating the information force. IEEE, vol. 1. IEEE, 2001, pp. 357361.

[9] M. Mansouri, A. Sardouk, L. Merghem-Boulahia, D. Gaiti, H. Snoussi, R. Rahim-Amoud, and C. Richard, "Factors that may influence the performance of wireless sensor networks," in Smart Wireless Sensor Networks. InTech, 2010 . 